\title{
Reconditioning of ti6al4v powder through an inductively coupled plasma for direct metal laser sintering
}

\author{
N Nkhasi ${ }^{1}, *$, W Du Preez ${ }^{2}$, H Bissett ${ }^{3}$ \\ ${ }^{1}$ Department of Mechanical and Mechatronics Engineering, Faculty of Engineering, Built Environment and Information Technology, Central \\ University of Technology, Free State, Bloemfontein, South Africa \\ ${ }^{2}$ Centre for Rapid Prototyping and Manufacturing, Faculty of Engineering, Built Environment and Information Technology, Central \\ University of Technology, Free State, Bloemfontein, South Africa \\ ${ }^{3}$ Applied Chemistry, South African Nuclear Energy Corporation SOC Ltd. (Necsa) Pretoria, South Africa \\ Email: ${ }^{1}$ thatengnp@gmail.com, ${ }^{2}$ wdupreez@cut.ac.za, ${ }^{3}$ hertzog.bissett@necsa.co.za
}

\begin{abstract}
Ti6Al4V is commonly used in the aerospace, medical and automotive industries due to its high strength-to-weight ratio and excellent corrosion resistance properties. Interstitial impurities in Ti6Al4V powder, particularly oxygen, nitrogen, hydrogen and iron, have an impact on mechanical properties. After Ti6Al4V powder has been re-used several times in direct metal laser sintering build processes, it will eventually reach its end of life due to its physical and chemical properties deteriorating out of specification. Such powder can be reconditioned using an inductively coupled plasma system. In this study, the reconditioning of the contaminated powder was done using an inductively coupled plasma system and characterisation of the Ti6Al4V powder was done before and after reconditioning. Conclusions are drawn on the feasibility of utilising an inductively coupled plasma system for reconditioning of contaminated Ti6Al4V powder.
\end{abstract}

Keywords: Direct metal laser sintering, Ti6Al4V powder, Inductively coupled plasma, Reconditioning.

\section{Introduction}

The direct metal laser sintering (DMLS) process, as referred to by ASTM F2792 - 12a (ASTM F2792 - 12a), like other powder bed fusion (PBF) processes, is challenged by low powder utilisation. In this process, the metal powder is spread over the substrate, and the laser fuses the new layer to the previously fabricated layer in a selective manner. The interaction between the high-energy laser and the metal powder bed can result in powder contamination through different phenomena including agglomeration, partial fusing, partial or full oxidation, metal vapour condensation and generation of spatter. After the desired part is fabricated, the used powder can either be discarded or recycled. Due to the high cost of metallic powders, specifically Ti6Al4V, powder recycling could be a viable option for cost reduction (Daraban et al. 2019, Carrion et al. 2019, O'Leary et al. 2015).

When dealing with Ti6Al4V powder, it must be kept in mind that the powder is more susceptible to oxygen pickup than other metals and, therefore, can only be used a few times before the powder falls out-of-specification due to the increase in oxygen content (Wei et al. 2017). To increase reusability of Ti6Al4V powder, it is important to identify qualified techniques to recondition out-ofspecification powder for use in PBF systems. One popular method is to blend virgin powder with the used powder before each build (Yan et al. 2020, Denti et al. 2019, Dastranjy Nezhadfar et al. 2018). This practice has produced large amounts of out-of-specification powder, either stored or wasted. All of these factors contribute to higher costs and a larger energy footprint of PBF processes. Another possible powder recycling method is using inductively coupled plasma (ICP) to recondition the metal powder because of its ability to produce a powder with an improved flowability, increased density and enhanced purity (Popovich and Sufiiarov 2016, Samant and Lewis 2017).

To keep PBF materials affordable as the technology expands, there is a need to develop qualified methods to recondition the powder and bring them back into production. There are a number of qualified techniques used for metal powder recycling to increase their reusability. Sartin et al. confirmed that metal additive manufacturing via laser PBF is challenged by low powder utilization and the ability to reuse metal powder will improve the process efficiency (Sartin et al. 2017). Samant and Lewis also studied metal powder recycling and reconditioning in PBF using Inconel 718 powder. Reconditioning of the metal powder was done using an induction plasma process and it was reported that reconditioning improved powder characteristics, such as flowability, by producing spherical particles and decreasing porosity by re-melting and re-solidifying the powder particles. Subsequently, the density was improved by improving powder purity through selective or reactive vaporisation of impurities (Samant and Lewis 2017).

In this study, the reconditioning of contaminated Ti6Al4V powder was done in an inductively coupled plasma (ICP) system. The "contaminated powder" is defined as the Ti6Al4V ELI (extra low interstitial) powder that has been previously used several times with its chemical composition subsequently not meeting the standard specification anymore. These interstitials are oxygen, nitrogen, hydrogen and iron. The alloy Ti6Al4V ELI is also known as titanium Grade 23. The purpose of the study was to determine 

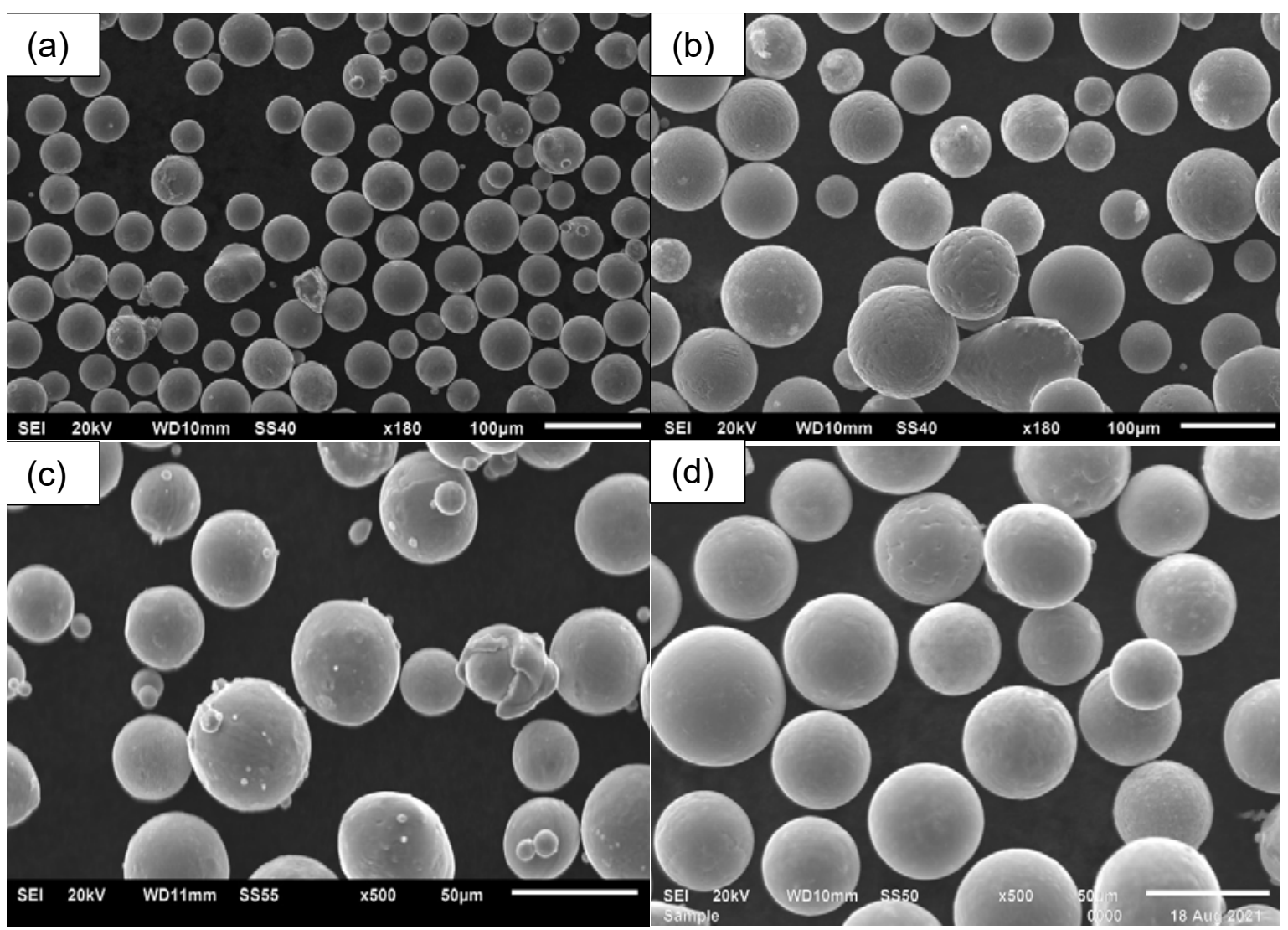

Figure 1: SEM SE micrographs micrographs of (a) and (c) contaminated Ti6Al4V powder and (b) and (d) reconditioned Ti6AL4V powder

whether the levels of interstitial elements could be reduced to prevent embrittlement, which is generally accepted as a problem in Ti6Al4V components produced through AM. The powder characterisation was done before and after reconditioning of the powder using different analytical techniques.

\section{Material and methods}

Contaminated Ti6Al4V powder supplied by the Centre for Rapid Prototyping and Manufacturing (CRPM) of the Central University of Technology, Free State (CUT) was used as feedstock for reconditioning in a $15 \mathrm{~kW}$ Tekna ICP system at the South African Nuclear Energy Corporation (NECSA). The particle size range of $38-45 \mu \mathrm{m}$ of contaminated Ti6Al4V powder was used as feedstock for plasma reconditioning. All powders were treated at a plasma plate power of $10 \mathrm{~kW}$ in argon thermal plasma at a reactor pressure of $85 \mathrm{kPa}$. Before removal of the powders from the Tekna system, the powders were passivated for safe removal. The resulting powders were subjected to a cleaning step to remove fines produced during the plasma treatment. The cleaning step was achieved by manually washing out the small particles that were not pulled away by the cyclone, using an ultrasonic process with water.

Characterisation of the as-received contaminated Ti6Al4V powder and reconditioned Ti6Al4V powder was done using different analytical techniques. The powders were characterised according to the ASTM F 3049 - 14 standard (ASTM F3049-14 2014). The morphology of both contaminated and reconditioned powder Ti6Al4V was determined using a high resolution JEOL JSM-6510 scanning electron microscope (SEM) with an energy dispersive
X-ray spectrometer (EDS). A Saturn DigiSizer II instrument was used to determine the Particle Size Distribution (PSD) and the porosity was determined through a high resolution General Electric Nanotom S X-ray micro-computed tomography (CT) system combined with detailed 3D data analysis at Stellenbosch University. The elemental composition of the powders was determined using a Spectro Arcos model inductively coupled plasma-optical emission spectroscopy (ICP-OES) instrument. A LECO ONH836 inert gas fusion analyser was used to determine the oxygen, nitrogen and hydrogen contents of the samples. Inert gas fusion was used in conjunction with ICP-OES to obtain a comprehensive determination of the chemistry of both contaminated and reconditioned powder.

\section{Results and discussion}

\subsection{Particle morphology}

In DMLS, it is well known that the particle morphology plays a significant role in powder performance including flowability and packing efficiency, and thus impacts the final component properties. Figure 1 shows representative SEM secondary electron (SE) micrographs for both contaminated and reconditioned Ti6Al4V powder.

Although many particles seem quite spherical, one can visually identify non-spherical particles, mainly caused by satellites (smaller particles being attached to larger particles) as shown in Figure 1(a) for the as-received contaminated Ti6Al4V powder. There were also single particles that were non-spherical without any satellites, as witnessed when the magnification was increased to $x 500$ in Figure 1(c). The latter could be due to melting of these 


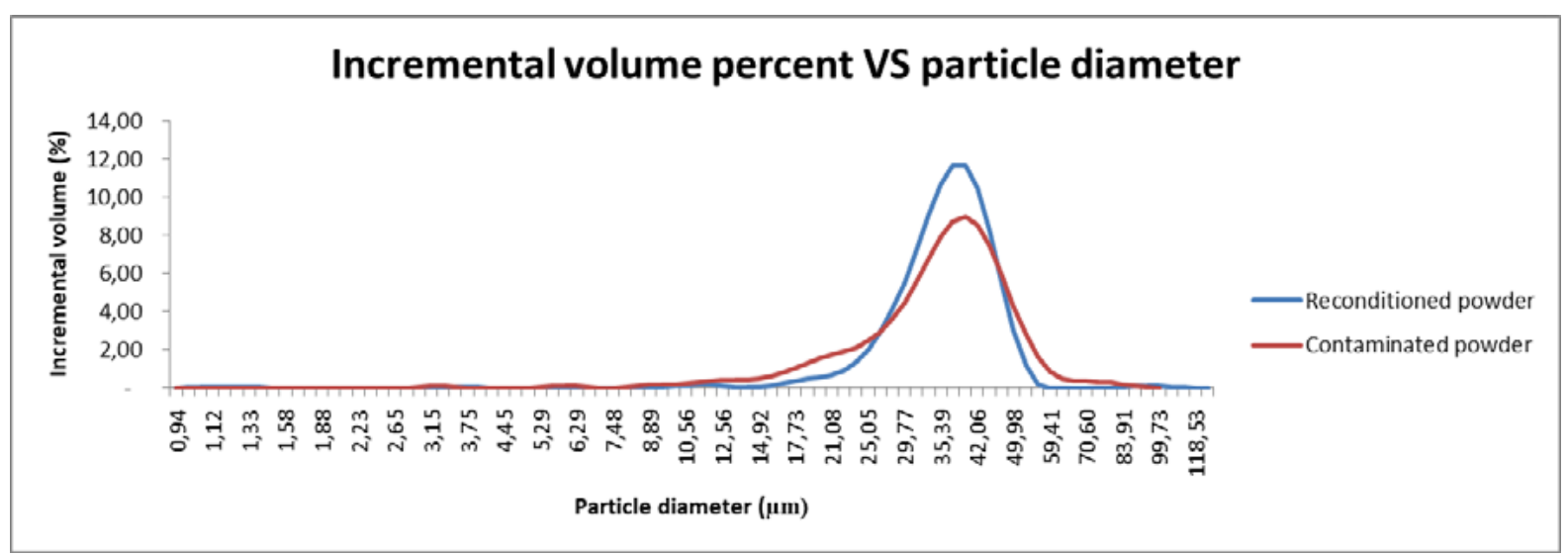

Figure 2: Particle size distribution of both contaminated and reconditioned Ti6Al4Vpowder

Table 1: Numerical PSD results for contaminated and reconditioned Ti6Al4V powder samples determined with the laser diffraction technique

\begin{tabular}{lcc}
\hline & Contaminated powder & Reconditioned powder \\
\hline $\mathrm{D}_{10}(\mu \mathrm{m})$ & 19.87 & 26.98 \\
$\mathrm{D}_{50}(\mu \mathrm{m})$ & 34.68 & 36.88 \\
$\mathrm{D}_{90}(\mu \mathrm{m})$ & 46.72 & 46.03 \\
\hline
\end{tabular}

particles during DMLS production cycles, because throughout the build process, many particles may be subjected to residual heat from the laser or melt pool when in proximity to the laser's targeted location. The presence of these non-spherical particles, categorised as agglomerates or partial melted individual particles, is likely to have an impact on inter-particle forces and packing density. This could be due to their different shapes interfering with the ability of particles to fit next to one another and pack tightly. These individual particle deformations are known to influence the overall behaviour of the powder. The micrograph in Figure 1(b) depicts the plasma treated particles that were spherical with no satellites. This can be explained as the result of particles being melted during plasma treatment while the small particles (satellites) attached to large particles were separated and formed two separate particles or melted together and formed one larger particle. At higher magnification of x650 (Figure 1(d)), which shows some of the large particles, the particles appeared to be perfectly spherical with no defects. Spherical particles are expected to increase the powder flow resulting in a homogeneous powder bed with high apparent density (Carrion et al. 2019).

\subsection{Particle size distribution (PSD)}

For the DMLS process, the determination of the PSD is essential, since this powder property has a large effect on the properties of the parts built, including the mechanical strength, porosity, and surface finish (Haferkamp et al. 2021). Figure 2 shows the PSD, as measured by laser diffraction, for contaminated and reconditioned Ti6Al4V powder. Comparing the two curves it is firstly interesting to note that both of them are in a state of normal distribution. Although the reconditioned Ti6Al4V powder has a higher peak than the contaminated Ti6Al4V powder, their peaks were at effectively the same particle diameter. The PSD curve of the reconditioned Ti6Al4V powder was narrower compared to the contaminated Ti6Al4V powder. Liu et al. also discovered that after plasma treatment the PSD curve of molybdenum had narrowed (Liu et al 2015).

Table 1 shows the values of $\mathrm{D}_{10}, \mathrm{D}_{50}$, and $\mathrm{D}_{90}$ for the contaminated and reconditioned Ti6Al4V powder. It is clear that the $\mathrm{D}_{10}$ and $\mathrm{D}_{50}$ values of the reconditioned Ti6Al4V powder are higher than that of the contaminated Ti6Al4V powder, which is in accordance with the SEM observations, as discussed in section 3.1.

In DMLS, the density of each layer should be as high as possible to reduce porosity in parts. One way to optimize powder bed density is through tailoring the PSD of a powder so that the smaller particles can fill the voids between the larger particles.
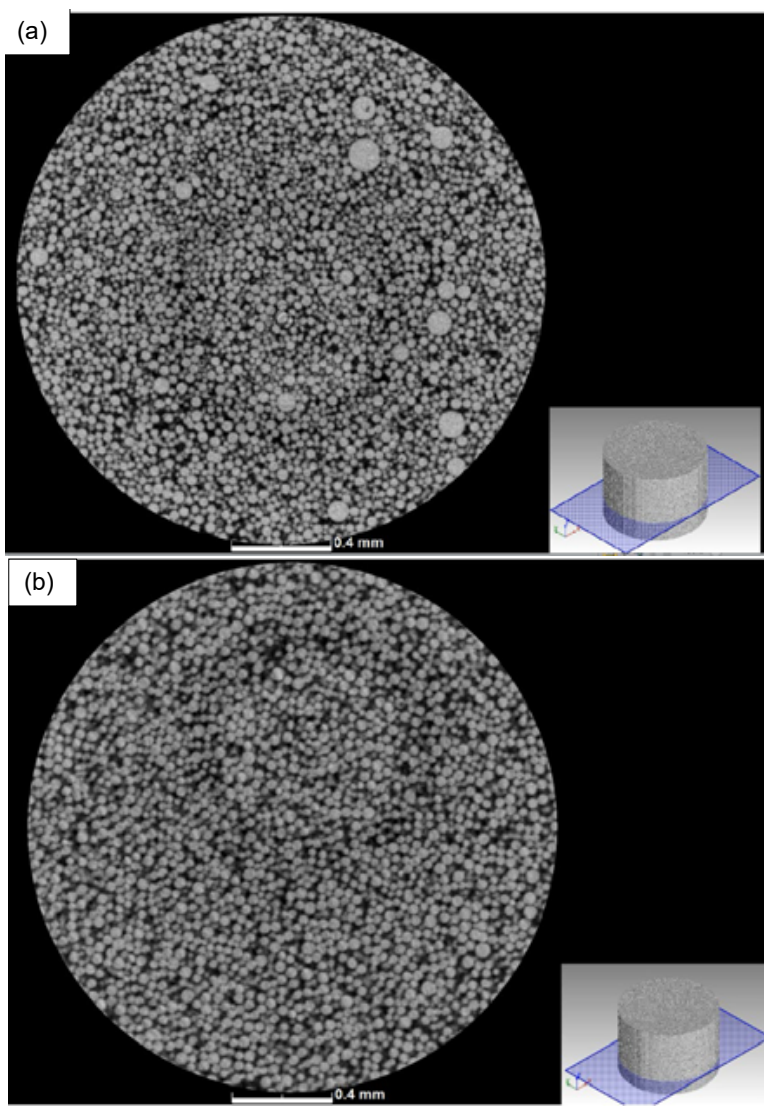

Figure 3: Micro-CT scan results showing slice images indicating pores in black inside the grey particles for (a) contaminated Ti6Al4V powder and (b) reconditioned Ti6Al4V powder 


\subsection{Particle porosity}

Samples of contaminated and reconditioned Ti6Al4V powders were analysed through micro-CT scanning to determine the powder particle porosity. The resultant slice images show internal porosity and details of the morphology. The internal porosity was detected as black areas inside grey particles, as shown in Figure 3. The contaminated Ti6A14V powder has some noticeable large particles with internal pores, as can be seen in the slice image in Figure 3(a). However, although internal pores could be detected in the particles of the reconditioned Ti6Al4V powder, they were not easily noticeable due to the even small particles of the reconditioned Ti6Al4V powder. This was due to the fact that the contaminated Ti6Al4V powder had totally melted during plasma treatment. In DMLS, the presence of excessive amounts of large pores or pores with entrapped gas can negatively affect material properties of parts produced through this process.

\subsection{Chemical composition}

The chemical composition of DMLS powder is an essential property, which has a determining effect on the chemical composition and mechanical properties of the final part. The ICP-OES results of the powder samples used in this study are given in Table 2. It could be noted that for contaminated Ti6Al4V powder, the concentrations of both aluminium and vanadium were already lower than the ASTM specified values. This could be due to as the melt pool undergoes a high degree of superheat, leads to vaporisation and material ejection as discussed by (Santecchia, Spigarelli, and Cabibbo 2020). During the DMLS process of Ti6Al4V, the element vaporisation is mainly controlled by the diffusion of the metal vapour in an inert atmosphere in the build chamber.

For aluminium, vanadium and iron the concentrations in the contaminated powder were out of specification. These lower levels need to be confirmed with further analyses to make sure it is consistently so. The concentrations of aluminium and iron were significantly lower in the reconditioned Ti6Al4V powder than in the contaminated Ti6Al4V powder. For aluminium, the change in concentration may be attributed to the sublimation of aluminium from the alloy, resulting in changing the chemical composition of the reconditioned Ti6Al4V powder during plasma treatment, because of the relatively low vaporisation temperature of aluminium (Zhang et al. 2020). From Table 2 it is clear that the iron content of the contaminated Ti6Al4V powder $(0.62 \mathrm{wt} \%)$ had increased to above the ASTM specified value of $0.25 \mathrm{wt} \%$. In a previous study (Thejane et al. 2017) this phenomenon was observed for reused Ti6Al4V (ELI) powder and was attributed to pickup of iron from the instruments used to handle and sieve the powder. Clearly, the reconditioning of the contaminated Ti6Al4V

Table 2: ICP-OES chemical composition results

\begin{tabular}{lcccc}
\hline Elements (wt (\%)) & Ti & Al & V & Fe \\
\hline $\begin{array}{l}\text { ASTM F3001 Ti6Al4V } \\
\text { (ELI) }\end{array}$ & 89 & $5.5-6.5$ & $3.5-4.5$ & 0.25 \\
Contaminated Ti6Al4V & 91.0 & 4.51 & 2.87 & 0.62 \\
Reconditioned Ti6Al4V & 91.8 & 4.17 & 2.80 & 0.22 \\
\hline
\end{tabular}

powder had the beneficial effect of reducing the iron content to within the specified level.

Oxygen, nitrogen and hydrogen contents of the powders are shown in Table 3 as determined by the LECO ONH836 inert gas fusion analyser. In contaminated Ti6 A14V powder the oxygen and nitrogen contents were found to still be within specification by (Thejane et al. 2017). This could be an indication that the inert argon atmosphere in the build chamber was successfully maintained at oxygen and nitrogen levels that were low enough to prevent diffusion of these gases into the built parts. The reconditioned Ti6Al4V powder has higher oxygen, nitrogen and hydrogen contents than the contaminated Ti6Al4V powder, however, the nitrogen and hydrogen contents still comply with the standard specification. It was also noted that the oxygen content was too high for Grade 5 titanium as specified by ASTM F1472 - 08 (ASTM F1472 - 08), as given in Table 3 .

Table 3: Oxygen, nitrogen and hydrogen content of the powders

\begin{tabular}{|c|c|c|c|}
\hline Sample & $\begin{array}{c}\text { Oxygen } \\
(\%)\end{array}$ & $\begin{array}{l}\text { Nitrogen } \\
\text { (\%) }\end{array}$ & $\begin{array}{l}\text { Hydrogen } \\
\text { (\%) }\end{array}$ \\
\hline $\begin{array}{l}\text { ASTM F3001 Ti6Al4V } \\
\text { (ELI) }\end{array}$ & 0.13 & 0.05 & 0.012 \\
\hline $\begin{array}{l}\text { ASTM F1472 - } 08 \\
\text { Ti6A14V }\end{array}$ & 0.2 & 0.05 & 0.015 \\
\hline Contaminated Ti6Al4V & 0.152 & 0.0203 & 0.00252 \\
\hline Reconditioned Ti6Al4V & 0.313 & 0.0269 & 0.01220 \\
\hline
\end{tabular}

With regard to DMLS, diffusion of oxygen into the metal to occupy interstitial positions, which could lead to formation of $\alpha$ phase, is viewed as detrimental and should be avoided due to its negative effect on the ductility of the alloy and potential to increase part porosity (Liu and Shin 2019). The challenge for Ti6Al4V powder treated in ICP is to produce Ti6Al4V powder with low oxygen. The impurity level and fine powder yield of ICP treated Ti6Al4V powder depend on the feedstock powder. Relative increased percentage of small particles might result in increased total surface area for oxygen layers to form on. For this reason small particles should be reduced either by changing the feedstock rate or power of the plasma system.

\section{Conclusion}

From this study it can be concluded that contaminated Ti6A14V powder can be successfully reconditioned in an inductively coupled plasma system to produce a homogeneous spherical powder. However, the chemical composition of the reconditioned Ti6Al4V powder does not fully meet the chemical composition as specified in the ASTM F3001-14 and ASTM F1472 - 08 standards. It is important to note that the contaminated Ti6Al4V powder was also already out of specification in terms of aluminium, vanadium, iron and oxygen. As comprehended from the results, lowering the oxygen and hydrogen content using an inductively coupled plasma system was a challenge. Therefore, further modification of the process parameters must be carried out to obtain an acceptable chemical composition. 


\section{Acknowledgments}

The authors would like to acknowledge the Nuclear Materials Development Network (NMDN) of the Advanced Materials Initiative (AMI), funded by the Department of Science and Innovation (DSI) for the financial support in conducting this study. The South African Nuclear Energy Corporation (Necsa) is acknowledged for their financial support. The following Necsa personnel are thanked for their contributions: Mr. MM Makhofane and Mr. PC Smith for the plasma experiments.

The financial support of the South African Department of Science and Technology through the Collaborative Program in Additive Manufacturing is gratefully acknowledged (Contract № CSIRNLC-CPAM-21-MOA-CUT-01).

\section{References}

1. ASTM F1472 - 08: Standard Specification for Wrought Titanium6Aluminum-4Vanadium Alloy for Surgical Implant Applications ( UNS R56400 ). 2008. 1-5.

2. ASTM F2792 - 12a: Standard Terminology for Additive Manufacturing Technologies. 2012. 1-3.

3. ASTM F3049-14: Standard Guide for Characterizing Properties of Metal Powders Used for Additive Manufacturing Processes. 2014. $1-3$.

4. Carrion P.E., Arash S., Nam P., and Nima S., 2019. Powder Recycling Effects on the Tensile and Fatigue Behavior of Additively Manufactured Ti-6Al-4V Parts. Journal of Materials Science 71(3), 963-73. https://doi.org/10.1007/s11837-018-3248-7.

5. Daraban A., Negrea, C.S., Artimon, F.G.P., Angelescu D., Popan G., Gheorghe S.I., Gheorghe M .2019. A Deep Look at Metal Additive Manufacturing Recycling and Use Tools for Sustainability Performance. Sustainability (Switzerland) 11(19) 1-20.
6. Dastranjy N., Pooriya S.T., Arash S., Amnda T., Nicolas S., 2018 Effects of Powder Recycling on the Mechanical Properties of Additively Manufactured Stainless Steel 17-4PH. Solid Freeform Fabrication Proceedings, 1292-1300.

7. Denti L., Antonella D., Silvio S., 2019. Effect of Powder Recycling in Laser-Based Powder Bed Fusion of Ti-6Al-4V. Manufacturing Technology 19(2), 190-96.

8. Haferkamp L., Haudenschild L., Spierings A.,Wegener K., Riener K., Ziegelmeier S., Leichtfried G., 2021. The Influence of Particle Shape, Powder Flowability, Bed Fusion. Metals 11, 1-14.

9. Liu X., Wang K., Hu P., Chan Q., Volisky A., 2015. Spheroidisation of Molybdenum powder by radio frequency thermal plasma. International Journal of Minerals, Metallurgy and Materials 22(11), 1212-1218.

10. O'Leary R., Setchi R., Prickett P., Hankins G., Jones N., 2015. An Investigation into the Recycling of Ti-6Al-4V Powder Used Within SLM to Improve Sustainability. The journal of Innovation Impact, 377-88.

11. Popovich A., Vadim S., 2016. Metal Powder Additive Manufacturing. $215-236$

12. Samant R., Brendan L., 2017. Metal Powder Recycling and Reconditioning in Additive Manufacturing. 1-2.

13. Santecchia E., Stefano S., and Marcello C., 2020. Material Reuse in Laser Powder Bed Fusion : Side Effects of the Laser - Metal Powder Interaction. Metals 10, 1-21.

14. Sartin B., Pond T., Griffith B., Everhart W., Elder L., Wenski E., Cook C., 2017. 316L Powder Reuse for Metal Additive Manufacturing. Solid Freeform Fabrication 2017: Proceedings of the 28th Annual International Solid Freeform Fabrication Symposium - An Additive Manufacturing Conference Reviewed Paper, 351-64.

15. Thejane K., Chikosha S, du Preez W.B, 2017. Characterisation and Monitoring of Ti6Al4V (ELI) Powder used in Different Selective Laser Melting Systems, South African Journal of Industrial Engineering, Special Edition, 28(3), pp 161-171.

16. Wei W., Wang L.Z., Chen T., Duan X.M., 2017. Study on the Flow Properties of Ti-6Al-4V Powders Prepared by Radio-Frequency Plasma Spheroidization. Advance Powder Technology 28, 2431-2437.

17. Yan Y., Chao Z.,Chao L., Mengzhi X., Ruihua Z., 2020. Effect of the Feeding Rate on Microstructure and Properties of Plasma Spheroidized GH4169 Powder. China Welding 29, 37-44. 